\title{
Implement Wireless and Distributed Vibrator for Enhancing Physical Activity of Visually Impaired Children
}

\author{
Hung-Chi Chu ${ }^{1,}$ Fang-Lin $\mathrm{Chao}^{*}, 2$, Liza Lee ${ }^{2}$, Pei-Yun $\mathrm{Kao}^{2}$ \\ ${ }^{1}$ Department of Information and Communication Engineering, Chaoyang University of Technology, 436, Taiwan R.O.C. \\ ${ }^{2}$ Department of Industrial Design, ${ }^{3}$ Department of Early Childhood Development \& Education, Chaoyang University of Technology, \\ 436, Taiwan R.O.C.
}

\author{
A R T I C L E I N F O \\ Article history: \\ Received: 27 October, 2019 \\ Accepted: 08 January, 2020 \\ Online: 15 January, 2020
}

Keywords:

Tactile sensation

Bodily activities

Visually impaired

Music

Wireless network

\begin{abstract}
A B S T R A C T
Learning and mental and physical counselling for visually impaired children are important. However, due to the lack of visual sense assistance, Physical Activity will gradually degenerate without proper enhancement, which may require the care of others for life. Inner fear and sensitive psychological factors hinder the development of living ability. Strengthening physical activity of visually impaired children improves their ability and reduces psychological obstacles, which is conducive to getting along well with others. The distributed vibration developed in this study can enhance children's physical exercise. Through music rhythm, children's psychological resistance is reduced, and their body movements are triggered by distributed vibration, which has an impact on the physical activity of children with visual impairment.

Seven kindergarten-age children were selected to participate in a 16-week tutoring. A score was recorded based on the assessment form, teaching record, and interviews with parents or students. The results showed that vibration and music are helpful in developing a young child's motor skills. After two sessions of tutoring, player became more interested in participating along with the music and tactile signals. Players were able to correctly identify the limbs that corresponded to the vibration and gesture with their hands and feet. Assistive technology had a positive effect on the physical activity of the visually impaired children.
\end{abstract}

\section{Introduction}

The daily life of the blind is not as active as ordinary people. The lack of visual sensory assistance results in problems such as slow movements and inability to respond immediately to the surrounding environment. Failure to integrate into normal life causes personality shrinkage, lack of contact with people, and decreased perception. With the advancement of technology, caring assistive technology has been applied to assist disadvantaged groups, such as: smart cane, route guidance, and voice/ image recognition. There were some facilities regarding to motor learning in visually impaired children [1,2,3]. Anthierens utilized a feel space navigation belt composed of 16 buzzers to distribute sensory cues for visually impaired kayakers [1]. Per Ellis presented sound therapy is effective for educating children who have multiple learning difficulties and may provide additional ways of communicating [4].

\footnotetext{
*Fang-Lin Chao, Email: flin@cyut.edu.tw
}

\subsection{Haptic Devices \& Teach}

Haptic devices which interact with the user without disturbing them are most commonly used for drawing the attention of cell phone owners. Small direct current (DC) motors offer high performance and a quick response time for a low price. Piezoceramics convert electrical energy into mechanical vibrations. Anvik [5] had investigated the potential of rich tactile notifications with six vibrators.

Previous studies have also shown positive effects through airflow and tactile sensations [6] in promoting children's physical activity. Baker et al. developed a 'ring' to be worn by an orchestral conductor and a 'vibration matrix' through a two-dimension haptic signal through which the conductor could convey their intent to a visually impaired musician [7]. Low-cost vibrators had also been constructed to promote general distribution of the application to disadvantaged group [8]. 


\section{H. Chu et al. / Advances in Science, Technology and Engineering Systems Journal Vol. 5, No. 1, 100-105 (2020)}

\subsection{Purpose}

The aim of this study was to examine the effect of distributed vibration in enhancing the motor control of visually impaired children with disabilities. Both quantitative and qualitative methods were utilized to measure the validity of the intervention. Next, six second-grade elementary school students participated in an activity which paired vibration events with song segments. During the session, players were able to correctly identify the limbs that corresponded to the vibration and gesture with their hands and feet.

Design actuators and tactile devices supported the effectiveness of distributed vibration in enhancing the movement of children. The specific purposes of the study were:

(1) To design affordable equipment for enhancing exercise behaviors.

(2) To examine the effectiveness of the equipment of distributed vibrator and music activities in enhancing physical activity in children.

\section{Assistive Device Design}

\subsection{Concepts and circuit implementation}

The initial concept art of the vibrator application is shown in Figure 1. This concept art proposed tiny distributed vibrators to prompt movement at specific positions on the user. Tactile signals cue the user to respond with an action at an individual location.

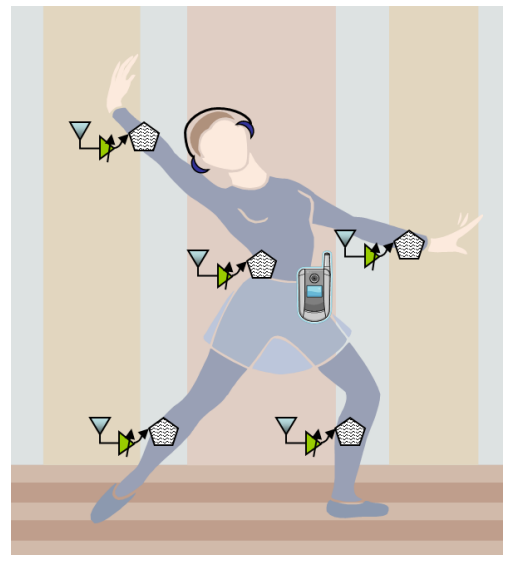

Figure 1: Concept art of distributed vibrators

For proper tactile cueing, the vibrators must contact the skin. In the early stage of the field test, we used elastic straps to attach the vibrator to the corresponding limb. Vibrators of the appropriate size received a signal through a wire; a computer controlled the on/ off timing of each channel.

The prototype's circuit consisted of solid-state relay (SSR) components through a USB line. The control channel employed a Phidget Interface Kit 8/8/8 I/O board, which controlled the switching by preset sequence. An external DC power supply with solid-state relay was constructed (Figure 2) with a $360 \mathrm{ohm}$ resistor in series with a $3.6 \mathrm{~V}$ Zener diode to ensure the current amount.

Figure 3 shows the graphical user interface (GUI) of the control program. The interface was developed using Microsoft Visual Basic, which includes five functions. The first part is the tool bar to provide the connection, COM part setup, Record, Stop, Execute, Camera, and Exit functions. The second part is the melody loading, and the third part is the load/modify function of body movements, which can load preconfigured body movements. Users can choose specific vibrator through push button: LH (left hand), RH (right hand), LF (left foot), and RF (right foot) buttons to active movements. Finally, parts four and five show the body movement results.

After selecting the music, users pressed the top button to begin. The time and order of the button pressing were recorded in a text file for playback during the next test.

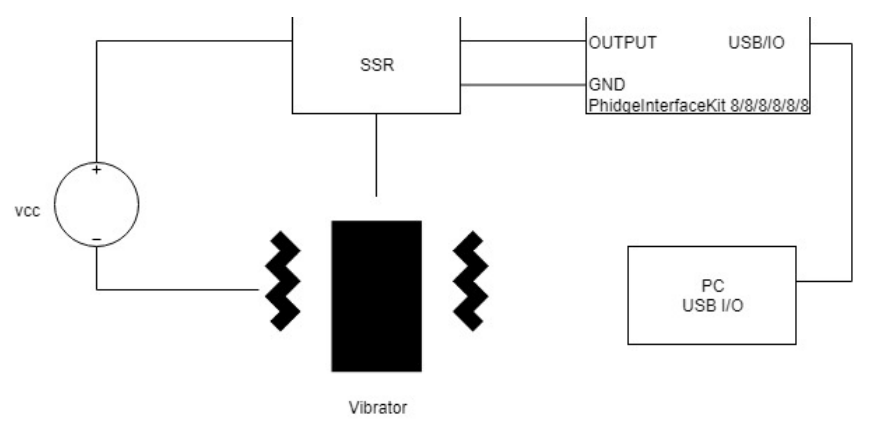

Figure 2: Hardware block diagram of the solid-state relay and vibrator

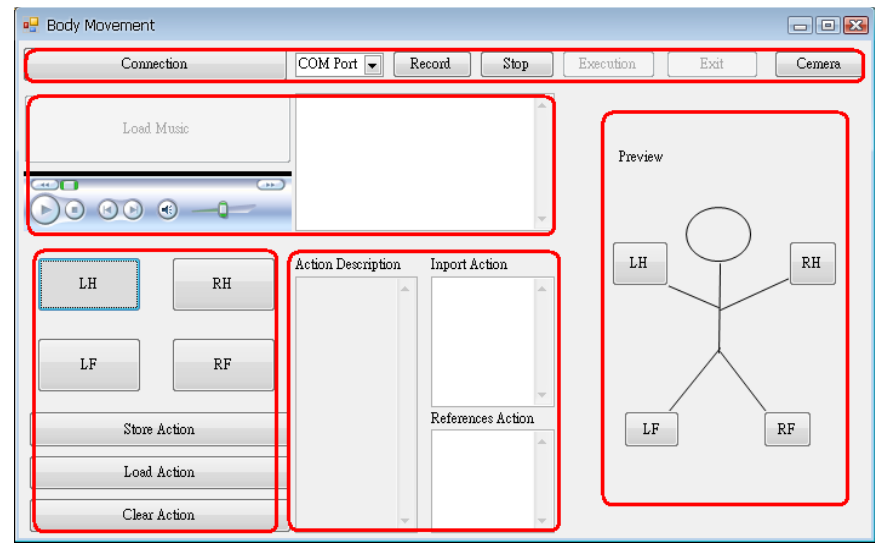

Figure 3: Editor screen layout of vibrators and control.

\subsection{Wireless WSN implementation}

In addition, with the rapid evolution of telecommunications technology, assistive technology combined with microelectronics and information technology has benefits. Wireless sensor network is an infrastructure less, low cost, dynamic topology, application oriented design with wireless distributed nods. WSN designing become more complex for further processing [9]. Deployed sensors provided the required connectivity [10]. Sensor and the wireless implementation of the distributed vibrator are reviewed in this section.

A WSN emphasizes low-cost, low-speed, and ubiquitous communication between devices. The basic framework involves a $10-\mathrm{m}$ communication range with a transfer rate of $250 \mathrm{kbps}$. The method significantly reduces line interference during movement. The system (Figure 4a) shows a central server using a ZigBee network interface, which is responsible for transmitting control signals and receiving messages from sensor nodes with the vibrator. The control command could turn on/off the vibrator with a specific time (Figure $4 \mathrm{~b}$ ). Each sensor module uses a $4.2 \mathrm{~V}$ lithium battery power. The vibrator is connected to a low-speed $\mathrm{I} / \mathrm{O}$ pin on the sensor node to reduce the module's energy 
consumption - the hardware circuit prototype placed within a chassis made by $3 \mathrm{D}$ printing (Figure 5). The ZigBee interface is IP-Link5501, and the sensor node is IP-Link1223 [11].

Zigbee's Network Layer Protocol Data Unit (NPDU) shows in the Figure 6. An NPDU consists of a network header and a network payload. The Network header contains frame control of 2 octets, routing fields of 6 octets, and data payload NSDU (network service data unit) of variable length. Besides, the routing fields of 6 octets are composed of a destination address of 2 octets, a source address of 2 octets, a radius (broadcast radius) of 1 octet, and a sequence number of 1 octet. Among them: the destination address of 2 octets is set to an address of 0xFFFF to indicate broadcast information, thereby broadcasting the control signals of the four vibrators at the same time.

The sequence number of 1 octet is the broadcast sequence number (BCSN). The control field of the vibrator used in this research is to use the 1 byte in the original data payload to mark it as ID1, ID2, ID3, and ID4. Each ID represents a controlled vibrator, and its field length is 2 bits, in which the bit value code: 11 means intensity vibration, 10 means moderate vibration, 01 means low vibration, and 00 means stop. ID1 and ID2 represent the left, righthanded vibrator, ID3 and ID4 represent the left, right-foot vibrator.

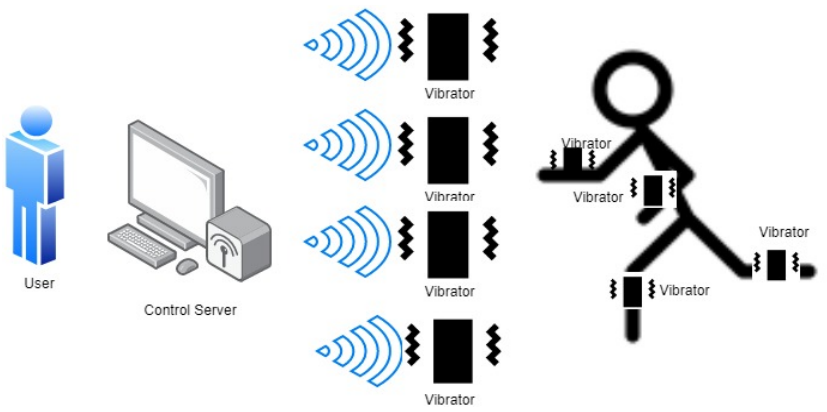

(a)

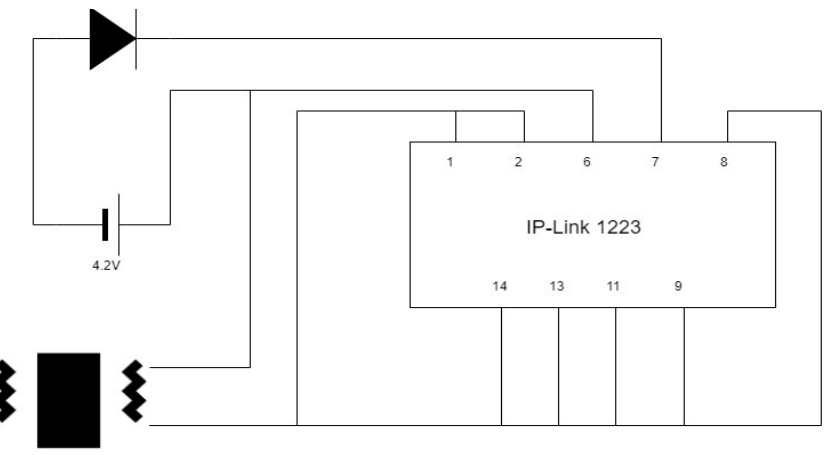

(b)

Figure 4: (a) Architecture of the assisted body movement system, (b) the circuit of vibration node.

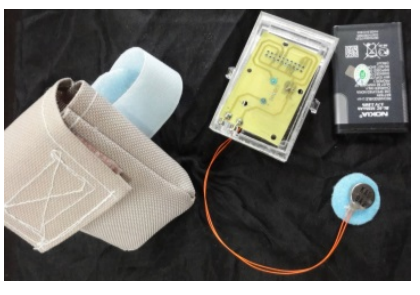

(a) (b)

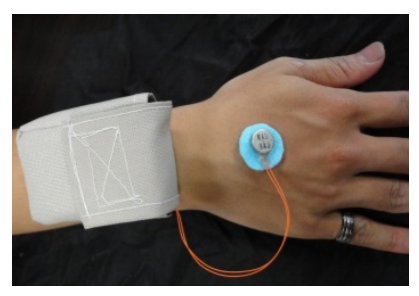

Figure 5: (a) Vibration node prototype with ZigBee Interface (IP-link 5501) and Sensor node: (IP-link 1223) (b) hand wearing.

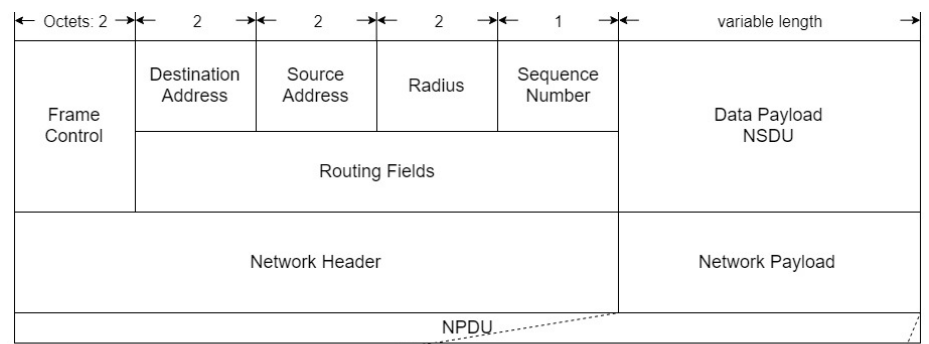

\begin{tabular}{|c|c|c|c|c|}
\hline $\mathrm{ID}_{1}$ & $\mathrm{ID}_{2}$ & $\mathrm{ID} 3$ & $\mathrm{ID}_{4}$ & Data Payload \\
\hline
\end{tabular}

Figure 6: Network Layer Protocol data unit of Zigbee.

The increase of the volume of the module weakens the vibration amplitude, which affects the perception and response of the subject. The designer tried EVA and placed vibrator under the main circuit with an eccentric shaft. The main circuit is separated from the round flat vibration motor. The arc shape conforms to the curvature of the wrist, and the fabric and the devil felt are connected for convenience (Figure 7).

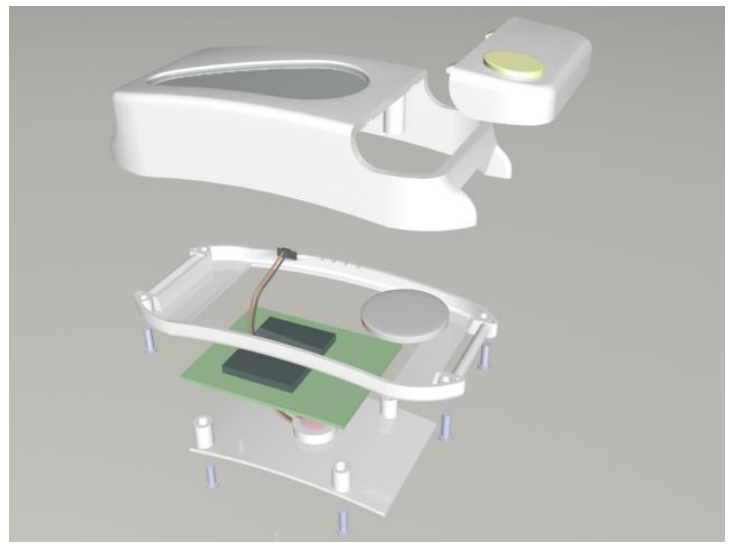

Figure 7: Module consists of an upper cover, chassis, and a battery cover. The chip is located on the lower chassis. There are power switch, mode setting button, and LED display switch.

\subsection{Human Factor issues}

Human factors design prompted ergonomic considerations for specific users. An interdisciplinary approach consist human factors, ergonomics, and work psychology in connection with design goals for cyber-physical product [12]. We kept the total weight of each vibrator under 130 grams through circuit miniaturization. The elastic straps followed the contours of the arm, wrist, or leg. Human factors were measured data. The maximum wrist circumference of the male wrist is about $19.8 \mathrm{~cm}$ and the average circumference of the adult ankle is about 25 centimeters. The initial design, featuring silicone material to help maintain skin contact with the backside-mounted vibrator, is shown in Figure 5.

The vibration amplitude/frequency need adjust for the specific user. Primary parameters of perception are: amplitude, frequency, timing, and location [13]. The skin is roughly sensitive to vibrations between 20 and $500 \mathrm{~Hz}$. Vibration stimuli exceeds threshold will be detected. The threshold for the trunk is 4 microns at $200 \mathrm{~Hz}[14,15]$. High spatial resolution located on the hands, and face. Tactile displays must be setting between threshold and the maximum comfort level. Comfortable stimuli range 15-20 dB 


\section{H. Chu et al. / Advances in Science, Technology and Engineering Systems Journal Vol. 5, No. 1, 100-105 (2020)}

above the absolute threshold. Amplitudes above 0.6-0.8 $\mathrm{mm}$ will result in a pain sensation. Design considerations reviewed considering: frequency, intensity, area of stimulation [16].

The stimulation responses of retarded children were analyzed in [17].Vibration sensation at low and high frequencies differed significantly. Sense thresholds lower frequencies (16 Hz and 31.5 $\mathrm{Hz}$ ) were low. The relatively unchanged tactile frequency discrimination between vibration frequencies of 180 and $250 \mathrm{~Hz}$ were measured in [18].The movement of the device limited to a narrow bandwidth of frequencies below $30 \mathrm{~Hz}$ [19].

Devices for locating or stimulating reflex points in the body with alternating magnetic fields producing oscillating movement which preferably exerting perpendicular forces on the skin [20]. Children's availability and movement convenience were prioritized for location choosing. When setting the vibrator, avoid high frequency or intensity to prevent tactile shock (Figure 8).

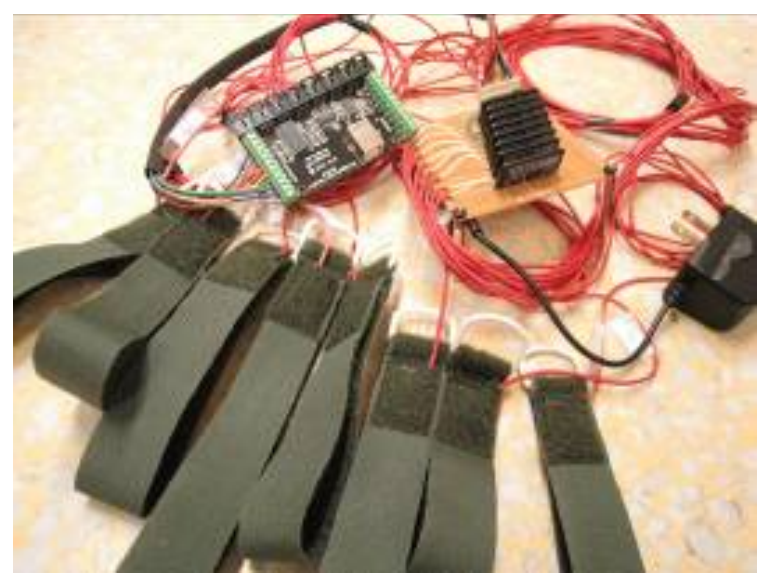

Figure 8: Wired module and external power supply.

\section{Methodology}

Visually impaired children have different athletic abilities with age and innate physical conditions. We have arranged tests for two ethnic groups:

(1) Children with multiple impairments of varying degrees of severity, aged between 3-6 years old, have limited ability to autonomously move. Due to the combined effects of other obstacles, the ability to move is limited, and the personality is closed and passive. Physical exercise encourages them to try to show more variety or amplitude.

(2) Visually impaired children have normal ability - aged 9-12. They are amblyopic or totally blind, but their physical capabilities are close to the average person. Close observation reveals that students have limited ability to balance, with small and restrained movements. Usually, they are accompanied by each other and passively do the activities within the allowable range under the teacher's request. Students may have obesity due to less activity. Physical exercise encourages them to demonstrate amplitude rhythm and interaction.

Based on the purpose of the study, the specific research questions asked were:

(1) Could multiple vibrators and music activities assist a young child with visually impaired development of motor skills?
(2) Could multiple vibrators enhance a visually impaired child's range of movement?

\subsection{Wired test}

The wired module is first introduced, it had limitation of connected wire but the facility cost is low and easy to implement. Teachers can give exercise tips in a small area. Two phases of testing were arranged to understand the adaptation to the vibrator for different ability: Phase- I involved group (1) 3-6 years-old with multiple disable, and Phase- II anticipate group (2) 9-12 years-old with normal ability.

Quantitative analysis utilized measuring validity based on assessment scales, semi-structured reports, and video recording. The therapeutic procedures included the establishment of goals, assessment, and activity evaluation. Some teaching styles were chosen based on participants' learning responses. The study took place at a school in central Taichung, Taiwan. Seven visually impaired participants were selected. Three participants exhibited severe physical disability, and the other four children exhibited moderate physical disability. The Phase-I participants were given 40-minute tutoring session once per week for 16 weeks (Figure 9).

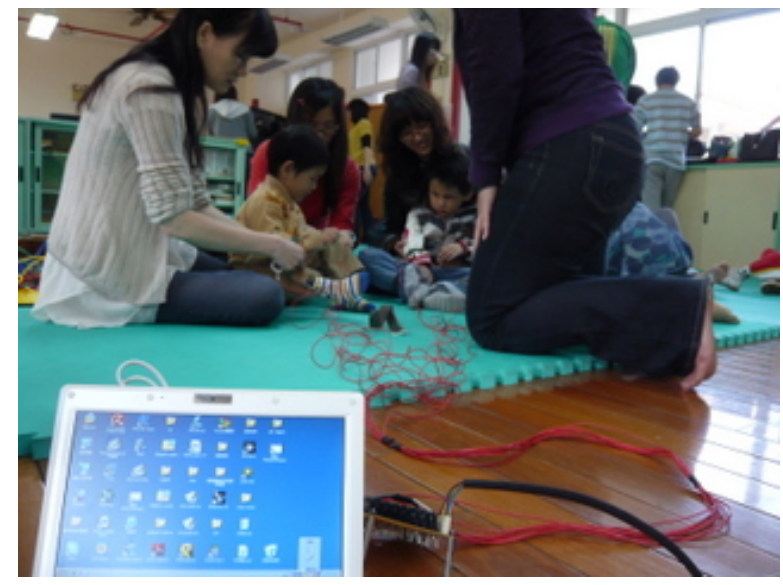

Figure 9: Field practice during Phase-I tutoring

The quantified results of a semi-structured musical activities observation form were compared with related background documents (reports from the parents and teaching logs). The forms were completed by a physical therapist in order to gather physical movement data which was scored by three observers.

\subsection{Wireless test}

The wireless module lifts the distance limitation of the vibration connection. In addition to prompting in a small range, you can also provide motion prompts in a large area covered by radio waves. The wireless module test also carried out to understand the adaptation to the vibrator for different ability group. The approaches were similar to wired test except utilized the wireless modules.

\section{Results and Discussion}

\subsection{Results of wired test}

\section{Phase- I Observation}

Figure 10 shows the participants' physical movements during the test. After attending the program, all participants exhibited significantly improved physical mobility. The three participants 
with severe symptoms were able to move their heads and hands slightly. The other four participants with moderate symptoms were able to walk and run stably and change direction. The tutoring sessions had a positive result (Figure 11).

Week 1 to Week 4: At the beginning of the research, none of the participants had the motivation to perform the movements. When the instructor put the distributed vibration equipment on the participants' limbs, and did not know what to do. The four participants with mild to moderate symptoms tried to pull the attached accessory. The frequency of the vibration was high and fast, consequently the participator could not follow it accordingly. A preset guidance protocol was required

Week 5 to Week 11 (same experiment setup): After the team adjusted the frequency of the vibration signal, most of the children did not reject the vibration and began to move to the music. Those with mild and moderate symptoms were able to move when participator felt the vibration and heard music. Sometimes participator would tell the instructor "felt the vibration on skin". The researchers found that the fuse of the attached accessory and cables was too long; as a result, the vibration signal did not transmit properly sometimes. Therefore, the team improved the cable routing and electrical contact.

Week 12 to Week 16: All the participants made progress at different levels (Figure 11). The participants with severe symptoms showed facial expressions of happiness. The participants with less severe symptoms were able to respond to the vibration and music correctly. The performance is slowly increasing by the time not right after using this equipment. For the mental state of the visually impaired with multiple obstacles, they couldn't accept changes from outside quickly. People with multiple disabilities have weak movements and expressions. After getting used to tactile sensations and being able to respond appropriately, additional channel of communication prompts them to participate more.
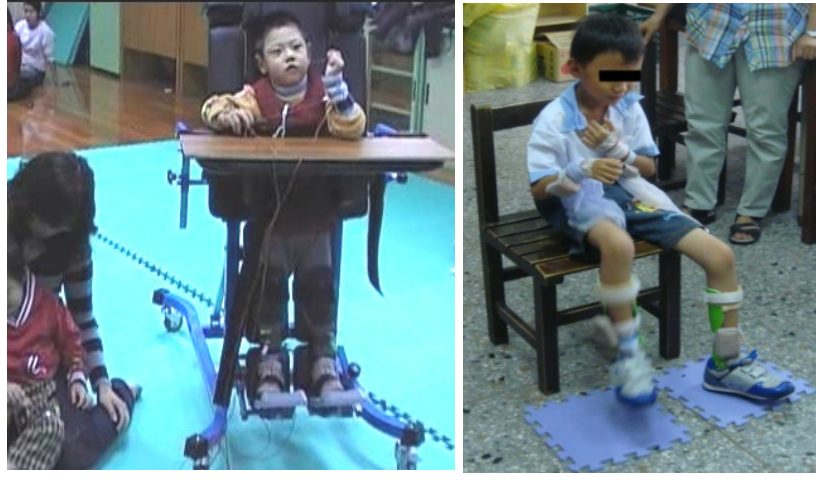

Figure 10: Participants' physical movement with (a) severe and (b) moderate symptoms

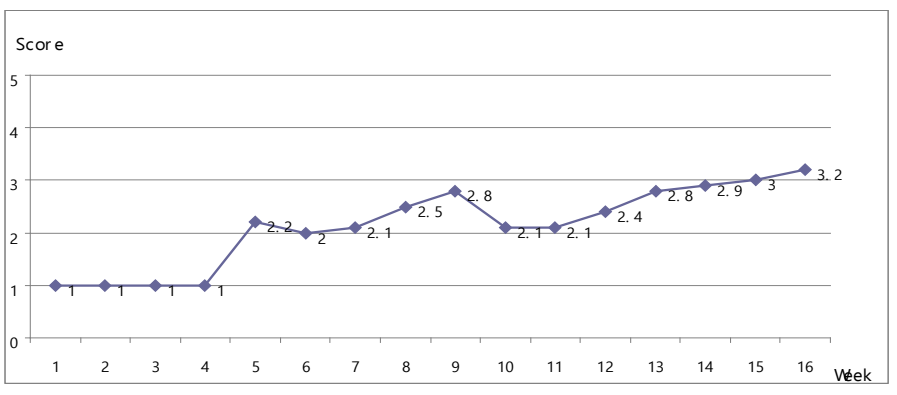

Figure 11: Observers' assessment of participants' physical movement

\section{Phase- II Observation}

Vibrators were placed on the upper and lower body (subjects' hands, wrists, and ankles). Six elementary school students participated in a five-minute session where vibration events were paired with segments of a song. The vibrators were turned on, along with the popular song, "Sorry," by Super Junier. Subject C01 (Figure 12a) appeared initially frightened and covered her ears. After the teacher's guidance, $\mathrm{C} 01$ was able to complete the directed actions.

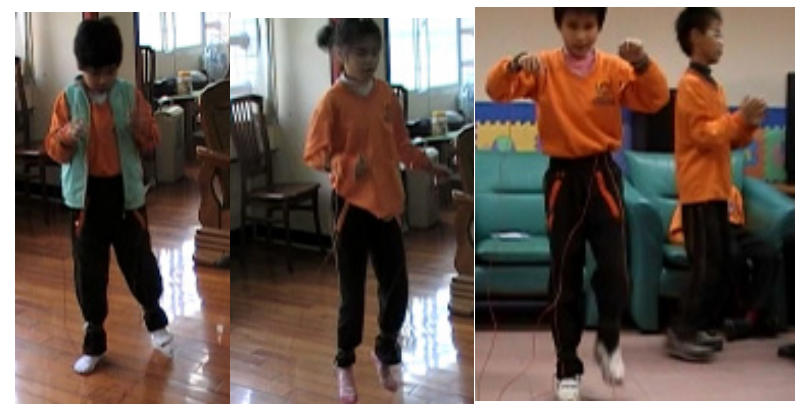

(a)

(b)

(c)

Figure 12: (a) C01's reaction, (b) C02, (c) C04/05situation

At first, Subject C02 (Figure 12b) found the experience strange and a little frightening. However, after guidance, $\mathrm{C} 02$ became more relaxed and willing to jump in place. Subject C03 swung her body around and occasionally moved her wrist in small motions. After some instruction, Subject $\mathrm{C} 03$ was able to wave her left arm. Subject C04 was more lively, swinging his body rhythmically and creating his own dance moves. He shook his body during the interlude and appeared to be having fun. Subject C05 (Figure 12c) could correctly identify which vibrator was vibrating and raise and lower his foot, but his rhythm was not as good as C04's. Both participants corresponded to the vibrations and made appropriate gestures with their hands and feet. Exercises make them more aware of their relative positions.

\subsection{Results of Wireless usage}

Ten special children are invited to perform small-scale sports, including children with low vision, blind children, and slow intellectual development. After instructing the child to equip the child with a vibrator, observe the response with the music and understand the differences in the way of using it.

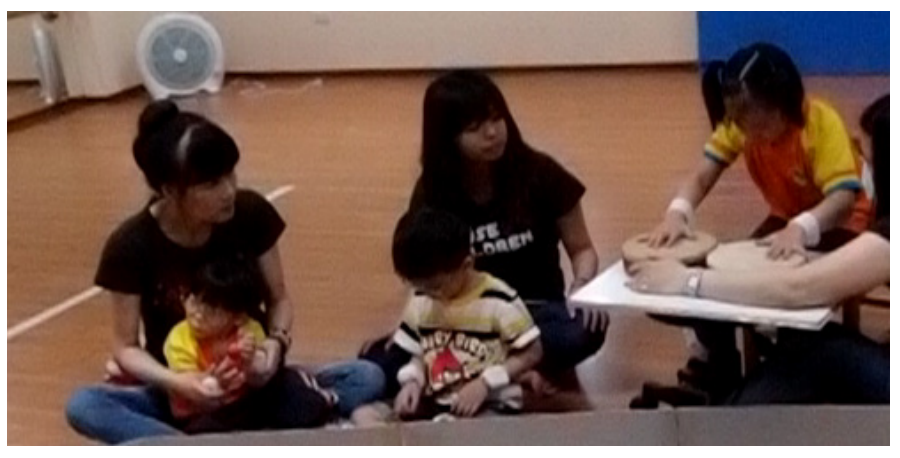

Figure 13: Children spontaneously tap the instrument

Children spontaneously tap the instrument according to the vibration instructions. Special children feel keen, and some children want to take off the vibrator because their hands are not comfortable. A child with a slow intellectual development has an absolute sensation of music show outstanding performance. Two 


\section{H. Chu et al. / Advances in Science, Technology and Engineering Systems Journal Vol. 5, No. 1, 100-105 (2020)}

student laugh and very focus on the indicator and tap the instrument. Some children complete the test through the guide of the accompanier. (Figure 13)

\section{Conclusions}

Through music activities with children of varying ages, the findings of the study were as follows: The participants' capacity for physical movement improved significantly. We also observed an improvement in social validity from the teachers and participants. The tactile design was able to assist the development of the participants' physical skill. The wireless module lifts the distance limitation of the vibration connection. In addition to prompting in a small range, you can also provide motion prompts in a large area covered by the radio waves.

Vibrator devices reinforce active participation for children with severe disabilities. Students exhibited high willingness to withstand new stimuli. Therefore, it is necessary to adapt a teaching strategy to the characteristics of the students. Wireless technology enables remote instructions to be given to assist users in interacting with the people and environment. This is not something that can be achieved by purely hardware design. The effectiveness of the equipment design also relies on developing the teaching strategy and events.

\section{Conflict of Interest}

The authors declare no conflict of interest.

\section{Acknowledgment}

This work was supported in part by the National Science Council, Taiwan, ROC, under grant NSC 101-2221-E-324-028, and 99-2221-E-324-026-MY2.

\section{References}

[1] Anthierens, Cédric, Didier Groux, and Vincent Hugel, "Sensory navigation guide for visually impaired sea kayakers" Journal of Field Robotics, 35(5), 732-747, 2018.

[2] Zuzanna Maćkowiak, Wieslaw Osiński,"The effect of sensorimotor training on the postural stability of visually impaired women over 50 years of age" Journal of Women \& Aging, 27(1), 68-80, 2015. DOI: $10.1080 / 08952841.2014 .928140$

[3] Malik, S. et al., "Orientation and mobility training in special education curriculum for social adjustment problems of visually impaired children in Pakistan" International Journal of Instruction, 11(2), 185-202, 2018.

[4] Phil Ellis, "Incidental music: A case study in the development of sound therapy," British Journal of Music Education, 12(1), 59-70, 1995.

[5] Cecilie H. Anvik, "Embodied spaces in the making: visually impaired people, bodies and surroundings" Scandinavian Journal of Disability Research, 11(2), 145-157, 2009. DOI: 10.1080/15017410902830710

[6] Schwellnus, H., et al. "Using movement-to-music technology for play with children with special needs" OT Now, July (2002).

[7] Baker, David, Ann Fomukong-Boden, and Sian Edwards, "'Don't follow them, look at me!': Contemplating a haptic digital prototype to bridge the conductor and visually impaired performer" Music Education Research 21(3), 295-314, 2019.

[8] Bischof, A. et al. "Exploring the playfulness of tools for co-designing smart connected devices: a case study with blind and visually impaired students" Proceedings of the 2016 Annual Symposium on Computer-Human Interaction in Play Companion,. ACM, 2016.

[9] Jangra, Ajay. "Wireless sensor network (WSN): Architectural design issues and challenges" 2010.

[10] Mohamed, Shaimaa M., Haitham S. Hamza, and Iman Aly Saroit, "Coverage in mobile wireless sensor networks (M-WSN): A survey" Computer Communications, 110, 133-150, 2017.

[11] IP-Link 1223, Helicomm Inc., http://www.helicomm.com/showproducts.asp?ID=144
[12] Stern, Hendrik, and Till Becker, "Development of a model for the integration of human factors in cyber-physical production systems" Procedia Manufacturing, 9, 151-158, 2017.

[13] Van Erp, J. B., "Guidelines for the use of vibro-tactile displays in human computer interaction" In Proceedings of eurohaptics, Vol. 2002, 18-22, 2002.

[14] Verrillo, R.T., "Effect of contactor area on the vibrotactile threshold" Journal of Acoustical Society of America, 35 (12), 1962-1966, 1963.

[15] Sherrick, C.A. \& Cholewiak, R.W., Cutaneous sensitivity. In Boff, K.R., Kaufman, L. \& Thomas, J.P.:Handbook of perception and human performance (pp. 12-1 -12-57), New York: John Wiley and sons, 1986.

[16] Verrillo, Ronald T. "Psychophysics of vibrotactile stimulation." The Journal of the Acoustical Society of America 77.1 (1985): 225-232.

[17] Pujol, Kelley Kruse. "The effect of vibrotactile stimulation, instrumentation, and precomposed melodies on physiological and behavioral responses of profoundly retarded children and adults" Journal of Music Therapy, 31(3), 186-205,1994.

[18] Harada, Noriaki, and Michael J. Griffin. "Factors influencing vibration sense thresholds used to assess occupational exposures to hand transmitted vibration" Occupational and Environmental Medicine, 48(3), 185-192, 1991.

[19] Wall, Steven A., and William Harwin. "A high bandwidth interface for haptic human computer interaction." Mechatronics 11.4 (2001): 371-387.

[20] Herman, Goldfarb. "Massaging garment with vibrators located in back and chest sections" U.S. Patent No. 3,310,050. 21 Mar. 1967. 